\title{
Quantum beat laser as a source of entangled radiation
}

\author{
Shahid Qamar, ${ }^{1, *}$ Fazal Ghafoor, ${ }^{1}$ Mark Hillery, ${ }^{2}$ and M. Suhail Zubairy ${ }^{1,3,4, \dagger}$ \\ ${ }^{1}$ Center for Quantum Physics, COMSATS Institute of Information Technology, Islamabad, Pakistan \\ ${ }^{2}$ Hunter College of CUNY, 65 Park Avenue, New York, New York 10065, USA \\ ${ }^{3}$ Institute for Quantum Studies and Department of Physics, Texas A \& M University, College Station, Texas 77843, USA \\ ${ }^{4}$ Texas A \& M University at Qatar, Education City, P. O. Box 23874, Doha, Qatar
}

(Received 17 January 2008; published 5 June 2008)

\begin{abstract}
We consider a quantum beat laser [Scully and Zubairy, Phys. Rev. A 35752 (1987)] as a source of entangled radiation. The system essentially consists of three-level atoms inside a doubly resonant cavity such that coherence is introduced by driving the upper two levels with a strong classical field of Rabi frequency $\Omega$. We study the dynamics of this system for different values of Rabi frequencies in the presence of cavity losses. It is shown that entanglement can be generated in this system for different initial states of the field in the two modes.
\end{abstract}

DOI: 10.1103/PhysRevA.77.062308

PACS number(s): 03.67.Mn, 03.67.Lx

\section{INTRODUCTION}

Due to the recent developments in the field of quantum computation, quantum communication, and quantum cryptography, interest has grown in the engineering of macroscopic entangled states of photons [1-3]. A number of different schemes have been proposed. These include the creation of entangled atomic ensembles through the transfer of a quantum state from nonclassical light to the atoms [4], the generation of bright two-mode quadrature squeezed light from a narrowband nondegenerate optical parametric amplifier, and many more [5-10]. Recently a scheme for the generation of bright entangled light using a correlated emission laser has also been proposed [11,12]. In further studies, the effects of phase and amplitude fluctuations associated with the driving field on entanglement generation were also studied $[13,14]$

In an earlier study, we considered a parametric converter as a source of entangled radiation [15]. We examined both the Duan, Giedke, Cirac, and Zoller (DGCZ) [16] and the Hillery-Zubairy [17] conditions for determining when the two modes in a parametric converter are entangled. It was shown that for different initial input states, the two criteria give different conditions that ensure that the output states are entangled.

In this paper, we consider the generation of entangled radiation by a quantum beat laser [18]. The system consists of three-level atoms in a V configuration interacting with two modes of the cavity field in a doubly resonant cavity. The atoms, which are pumped incoherently in their excited state, are driven into a coherent superposition of the upper two levels by a strong classical field. It was shown that this system exhibits the vanishing of the phase diffusion in the relative phase of the two modes under certain conditions [18]. In some earlier studies, the experimental observation of the

\footnotetext{
*Permanent address: Department of Physics and Applied Mathematics, Pakistan Institute of Engineering and Applied Sciences, Nilore, Islamabad, Pakistan. shahid_qamar@pieas.edu.pk

†zubairy@physics.tamu.edu
}

noise reduction in the relative phase of a two-mode laser has also been discussed [19]. In order to estimate the entanglement in a quantum beat laser, we consider the recently proposed conditions by Simon [20], DGCZ [16], and Hillery and Zubairy [17]. We consider different initial states for the two-mode field and discuss the temporal evolution of the field for different values of the Rabi frequency $\Omega$ associated with the classical field. Our results show that two modes, which are initially in a product state, evolve into an entangled state even in the presence of cavity losses. However, the time for which the two modes remain entangled strongly depends upon the Rabi frequency $\Omega$. An increase in the strength of the driving field causes the time for which the output field is entangled to increase. This result is in agreement with our earlier study of the generation of entanglement in a correlated spontaneous emission laser [11]. Our results also show that the two entanglement criteria give different time intervals for which the two-mode field remains entangled.

\section{THE SYSTEM}

In this section, we consider the atomic system for the quantum beat laser proposed by Scully and Zubairy [18], which is shown in Fig. 1. It is a three-level atomic system in a $\mathrm{V}$ configuration. The atoms are pumped into the state $|a\rangle$ at a rate $r_{a}$. A doubly resonant cavity containing two modes is considered. The transitions between levels $|a\rangle$ and $|c\rangle$ and $|b\rangle$ and $|c\rangle$ are dipole allowed while the transition $|a\rangle-|b\rangle$ are electric-dipole forbidden, but can be induced by applying a strong magnetic field for a magnetic-dipole-allowed transition. The transition $|a\rangle-|b\rangle$ is treated semiclassically to all orders in the Rabi frequency. The transitions $|a\rangle-|c\rangle$ and $|b\rangle-|c\rangle$ are treated fully quantum mechanically but only up to second order in the corresponding coupling constants. The Hamiltonian in the dipole and rotating wave approximations is given by

$$
\begin{gathered}
H=H_{0}+V, \\
H_{0}=\sum_{i=a, b, c} \hbar \omega_{i}|i\rangle\langle i|+\hbar \nu_{1} a_{1}^{\dagger} a_{1}+\hbar \nu_{2} a_{2}^{\dagger} a_{2},
\end{gathered}
$$




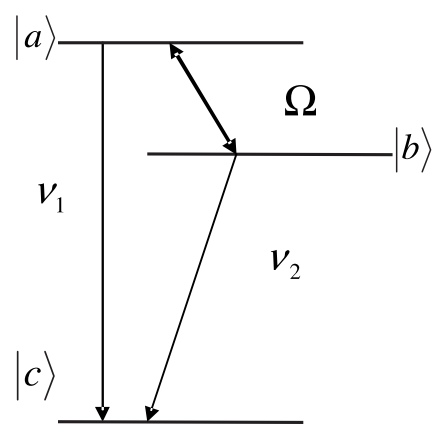

FIG. 1. Energy level diagram for the quantum beat laser, a threelevel atomic system in a $\mathrm{V}$ configuration. The transitions between levels $|a\rangle$ and $|c\rangle$ and $|b\rangle$ and $|c\rangle$ at frequencies $\nu_{1}$ and $\nu_{2}$ are resonant with the cavity. The transition $|a\rangle-|b\rangle$ is electric-dipole forbidden but can be induced by a strong magnetic field if it is magnetic-dipole allowed.

$$
\begin{aligned}
V= & \hbar g_{1}\left(a_{1}|a\rangle\left\langle c\left|+a_{1}^{\dagger}\right| c\right\rangle\langle a|\right)+\hbar g_{2}\left(a_{2}|b\rangle\left\langle c\left|+a_{2}^{\dagger}\right| c\right\rangle\langle b|\right) \\
& -\frac{\hbar \Omega}{2}\left(e^{-i \phi-i \nu_{3} t}|a\rangle\left\langle b\left|+e^{i \phi+i \nu_{3} t}\right| b\right\rangle\langle a|\right)
\end{aligned}
$$

where $a_{1}\left(a_{1}^{\dagger}\right)$, and $a_{2}\left(a_{2}^{\dagger}\right)$ are the annihilation (creation) operators for the fields in the two modes, whose frequencies are $\nu_{1}$ and $\nu_{2}, g_{1}\left(g_{2}\right)$ is the coupling constant associated with the $|a\rangle-|c\rangle(|b\rangle-|c\rangle)$ transition; and $\nu_{3}$ is the frequency of the field that induces the transition between levels $|a\rangle$ and $|b\rangle$, which is assumed to be at resonance with the $|a\rangle-|b\rangle$ transition, i.e., $\nu_{3}=\omega_{a}-\omega_{b}$. The equation of motion for the field density matrix is given by

$$
\begin{aligned}
\dot{\rho}_{f}= & -\frac{1}{2}\left[\alpha_{11}^{*} a_{1} a_{1}^{\dagger} \rho_{f}+\alpha_{11} \rho_{f} a_{1} a_{1}^{\dagger}-\left(\alpha_{11}+\alpha_{11}^{*}\right) a_{1}^{\dagger} \rho_{f} a_{1}\right] \\
& -\frac{1}{2}\left[\alpha_{22}^{*} a_{2} a_{2}^{\dagger} \rho_{f}+\alpha_{22} \rho_{f} a_{2} a_{2}^{\dagger}-\left(\alpha_{22}+\alpha_{22}^{*}\right) a_{2}^{\dagger} \rho_{f} a_{2}\right] \\
& -\frac{1}{2}\left[\alpha_{21}^{*} a_{1}^{\dagger} a_{2} \rho_{f}+\alpha_{12} \rho_{f} a_{1}^{\dagger} a_{2}-\left(\alpha_{12}+\alpha_{21}^{*}\right) a_{1}^{\dagger} \rho_{f} a_{2}\right] e^{i \Phi} \\
& -\frac{1}{2}\left[\alpha_{12}^{*} a_{1} a_{2}^{\dagger} \rho_{f}+\alpha_{21} \rho_{f} a_{1} a_{2}^{\dagger}-\left(\alpha_{21}+\alpha_{12}^{*}\right) a_{2}^{\dagger} \rho_{f} a_{1}\right] e^{-i \Phi} \\
& -\kappa_{1}\left(a_{1}^{\dagger} a_{1} \rho_{f}-2 a_{1} \rho_{f} a_{1}^{\dagger}+\rho_{f} a_{1}^{\dagger} a_{1}\right) \\
& -\kappa_{2}\left(a_{2}^{\dagger} a_{2} \rho_{f}-2 a_{2} \rho_{f} a_{2}^{\dagger}+\rho_{f} a_{2}^{\dagger} a_{2}\right),
\end{aligned}
$$

where we include the cavity damping terms in the usual way. It is assumed that the two cavity modes are coupled to two independent vacuum reservoirs with $\kappa_{1}$ and $\kappa_{2}$ being the cavity decay rates of modes 1 and 2 , respectively. Here the terms proportional to $\alpha_{11}$ and $\alpha_{22}$ are the gain terms, and the terms proportional to $\alpha_{12}$, and $\alpha_{21}$, are the phase-sensitive terms that are generated due to the atomic coherence produced between the levels $|a\rangle$ and $|b\rangle$ by the strong driving field. Explicit expressions for the coefficients $\alpha_{11}, \alpha_{12}, \alpha_{21}$, and $\alpha_{22}$ in Eq. (4) are given in Appendix A. The phase angle $\Phi$ appearing in Eq. (4) equals $\Phi=\phi+\left(\nu_{1}-\nu_{2}-\nu_{3}\right) t$. In our analysis we consider exact resonance under that condition $\Phi=\phi$, which is the external field reference phase.

\section{ENTANGLEMENT CONDITIONS FOR TWO-MODE STATES}

The existence and estimation of entanglement for a mixed state remains an important question [17]. A state is entangled if it is not separable. A state is separable if its density matrix $\rho$ can be expressed as a convex combination of product states,

$$
\rho=\sum_{j} p_{j} \rho^{(1)} \otimes \rho_{j}^{(2)}
$$

with $p_{j} \geq 0$ and $\Sigma_{j} p_{j}=1$. If it cannot be expressed in this form, it is entangled. For a pure state there exist sufficient and necessary conditions for bipartite entanglement, while for an arbitrary mixed state there exists no sufficient and necessary criterion for the existence of entanglement. However, in some recent studies several sufficient criteria for entanglement have been proposed [21-24]. One of the most extensively used is the Peres-Horodecki criterion, which is based on the fact that, if a state is separable, then the partial transpose of its density matrix must be positive $[23,24]$. Similarly, DGCZ [16] and independently Simon [20] proposed a class of conditions that is sufficient to show entanglement in continuous-variable systems, which involves only low-order moments of the mode quadrature operators. In fact, for Gaussian states, these conditions are necessary and sufficient to show entanglement. More recently, Hillery and Zubairy [17] found sufficiency conditions for entanglement for a different class of states. They provide a class of inequalities whose violation shows the presence of entanglement in a two-mode system. These methods can be extended to find conditions for entanglement in systems consisting more than two modes. In a recent study, we applied these conditions to study the generation of entanglement in several devices [25].

In order to study the generation of entanglement in a quantum beat laser, we shall first consider the HilleryZubairy criterion, which states that a state is entangled if [17]

$$
\left|\left\langle a_{1} a_{2}^{\dagger}\right\rangle\right|^{2}>\left\langle N_{1} N_{2}\right\rangle
$$

where $N_{1}=a_{1}^{\dagger} a_{1}$, and $N_{2}=a_{2}^{\dagger} a_{2}$ are the photon number operators in modes 1 and 2 , respectively. Here we are interested in studying how the generation of entanglement depends on the Rabi frequency $\Omega$ associated with the driving field. Since the exact analytical results are rather complicated in this situation, we shall present the results of our numerical simulations. The equations of motion for various moments that are required to evaluate the quantities involved in Eq. (6) are given in Appendix B.

In the parametric limit, when the Rabi frequency is much larger than the atomic decay rate $\gamma$, i.e., $\Omega \gg \gamma$, the coefficients $\alpha_{11}$ and $\alpha_{22}$ which are given by Eqs. (A1) and (A4) in Appendix A approach zero. Under this condition, the equation of motion for the density matrix given by Eq. (4) approaches the equation of motion for a parametric converter. In a recent study, we discussed the generation of entanglement in a parametric converter using the DGCZ [16] and the 


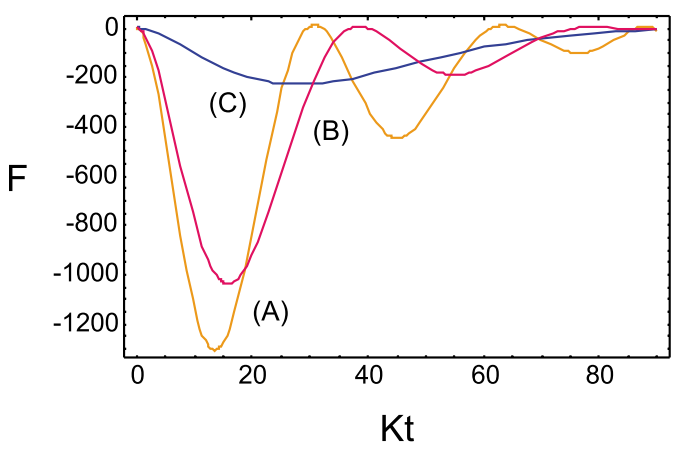

FIG. 2. (Color online) Time development of $F \equiv\left\langle N_{1} N_{2}\right\rangle$ $-\left|\left\langle a_{1} a_{2}^{\dagger}\right\rangle\right|^{2}$ for initial squeezed vacuum state in mode $a$ and coherent state in mode $b$ with $r=0.1$ and $\alpha=10$ (in terms of normalized interaction time $K t$ ). Various parameters are $\gamma=20 \mathrm{kHz}, \kappa_{1}=\kappa_{2}$ $=1.5 \mathrm{kHz}, \mathrm{g}=43 \mathrm{kHz}$, and $r_{a}=22 \mathrm{kHz}$. Here curves (A), (B), and (C) represent the results for $\Omega=400,500$, and $1500 \mathrm{kHz}$, respectively.

Hillery-Zubairy [17] criteria, and obtained the conditions on the input modes that ensure that the output modes are entangled.

\section{NUMERICAL RESULTS}

Here we present the results of our numerical simulation. In Fig. 2, we show the plot of the time development of $F$ $\equiv\left\langle N_{1} N_{2}\right\rangle-\left|\left\langle a_{1} a_{2}^{\dagger}\right\rangle\right|^{2}$ versus the dimensionless interaction time $K t$. The parameter $K$ is defined as $K=g^{2} r_{a} / \gamma^{2}$. We consider the initial squeezed vacuum state in mode 1 , which can be represented as $S(r)|0\rangle$ with the squeezing operator $S(r)$ $=\exp \left[(r / 2)\left(a^{2}-a^{\dagger^{2}}\right)\right]$ defined in terms of the creation and annihilation operators. The parameter values are chosen such that they correspond to the micromaser experiment of $\mathrm{Me}-$ schede et al. [27]. It may be mentioned that the system we are discussing here can be realized by placing the gain medium inside a doubly resonant cavity. We can also consider the system in which atoms with long-lived states pass through the cavity such that there is only one atom at a time inside the cavity in the presence of classical driving fields. This corresponds to the experimental setup used in the micromaser $[27,28]$. It is clear from the curves that the parameter $F$ becomes negative as time evolves, which shows that the two modes, which are initially in a product state, evolve into an entangled state. For $\Omega=400$ and $500 \mathrm{kHz}$, oscillations appear whose amplitudes decrease due to the presence of cavity losses. During the evolution, there is a time at which $F$ becomes zero, and the entanglement criterion is no longer satisfied. Our results show that the time for which the two modes remain entangled strongly depends upon the strength of the Rabi frequency. When we further increase the value of $\Omega$, such that it is equal to $1500 \mathrm{kHz}$, then the entanglement time increases further. This behavior is in agreement with our earlier study, which dealt with the generation of entanglement in a correlated spontaneous emission laser [11].

In order to get some insight, next we show the time development of average photon numbers in modes 1 and 2 for the same set of parameters as in Fig. 2. The average photon
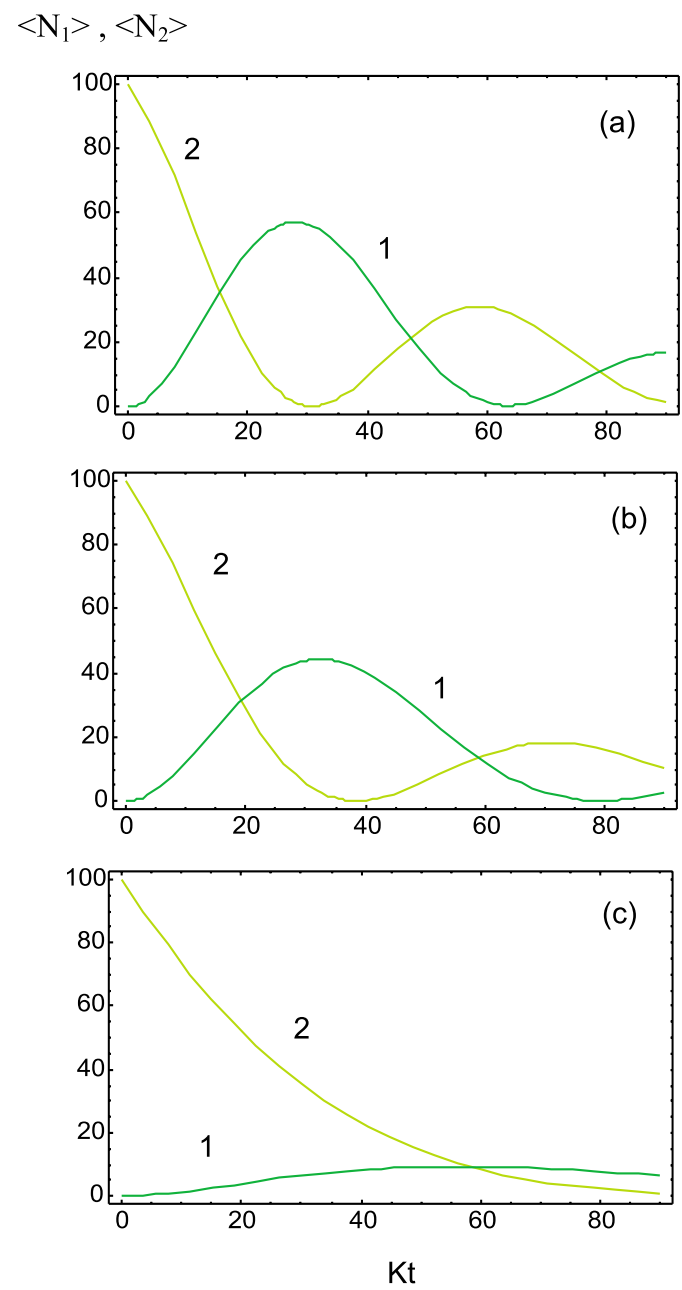

FIG. 3. (Color online) Time development of average photon numbers $\left\langle N_{1}\right\rangle$ and $\left\langle N_{2}\right\rangle$ against the normalized interaction time $K t$ for (a) $\Omega=400$, (b) 500 , and (c) $1500 \mathrm{kHz}$, respectively. Here curve 1 corresponds to mode 1 and curve 2 to mode 2 . All the parameters are the same as in Fig. 2.

numbers evolve with time and exhibit oscillatory behavior in Figs. 3(a) and 3(b), which correspond to $\Omega=400$ and 500 $\mathrm{kHz}$. However, the amplitude of oscillations decreases, due to the presence of cavity losses. During the oscillations, there are times at which the average photon numbers become zero. Note that, because it is the correlation between the two modes that gives rise to entanglement in a quantum beat laser, when the average photon number in one of the modes vanishes, then the correlation no longer exists and the entanglement criterion is no longer satisfied. This is clear from Fig. 3. For $\Omega=1500 \mathrm{kHz}$, the average photon number remains nonzero for the time interval shown in Fig. 2, and, as a result, correlations exist that lead to entanglement. A comparison of Figs. 2 and 3 clearly shows that, due to the increase in the Rabi frequency, the time period for which the photon numbers in the two modes remain nonzero increases, and this gives rise to an increase in the entanglement time.

In Fig. 4, we show the results of our numerical simulation for the case when mode 1 is initially in a photon number state with ten photons and mode 2 is in a vacuum state. All the other parameters are the same as in Fig. 2. The results 


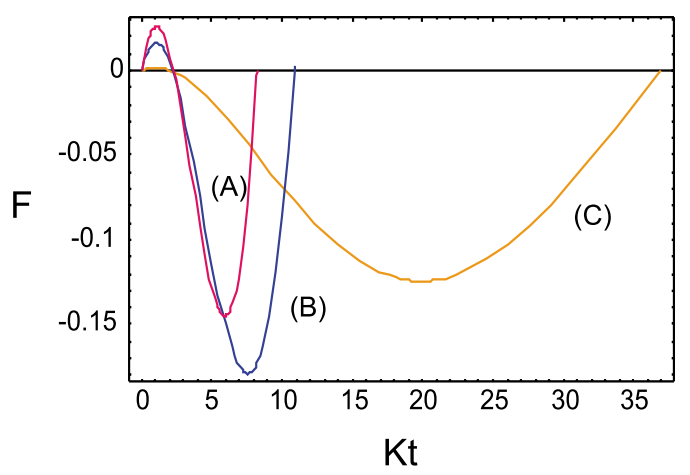

FIG. 4. (Color online) Time development of $F \equiv\left\langle N_{1} N_{2}\right\rangle$ $-\left|\left\langle a_{1} a_{2}^{\dagger}\right\rangle\right|^{2}$ for a photon number state with ten photons in mode 1 and the vacuum state in mode 2 . All the other parameters are the same as in Fig. 2.

clearly show that, as the parameter $F$ evolves with time, it becomes negative. Here the curves are truncated at the point where the value of $F$ becomes equal to zero. Our results show that the system evolves into an entangled state and remains entangled for a period of time. The curves (A), (B), and $(\mathrm{C})$, which correspond to different values of Rabi frequency, again show that the time for the entanglement is not the same, and that it increases as the Rabi frequency $\Omega$ of the driving field increases.

\section{SIMON-DGCZ ENTANGLEMENT CRITERION FOR TWO-MODE STATES}

In this section we consider the Simon-DGCZ entanglement criterion to study the generation of entanglement in a quantum beat laser. According to this criterion, a state of the system is known to be entangled if the quantum fluctuations of the two Einstein-Podolsky-Rosen-like operators $\hat{u}$ and $\hat{v}$ of the two modes satisfy the following inequality:

$$
(\Delta \hat{u})^{2}+(\Delta \hat{v})^{2}<2 .
$$

This inequality gives a sufficient condition for the entanglement of a two-mode state. It is one of the class of entanglement criteria found by Duan et al. and by Simon $[16,20]$. In Eq. (7),

$$
\begin{aligned}
& \hat{u}=\hat{x}_{1}+\hat{x}_{2}, \\
& \hat{v}=\hat{p}_{1}-\hat{p}_{2} .
\end{aligned}
$$

and $x_{j}=\left(a_{j}+a_{j}^{\dagger}\right) / \sqrt{2}$ and $p_{j}=\left(a_{j}-a_{j}^{\dagger}\right) / \sqrt{2}$ (with $\left.j=1,2\right)$ are the quadratures for the two modes of the cavity field. These can be measured in an experiment by using the technique of balanced homodyne detection [26]. If we substitute the definition of $\hat{u}$ and $\hat{v}$ in Eq. (7), we obtain

$$
\begin{aligned}
(\Delta \hat{u})^{2}+(\Delta \hat{v})^{2}= & \left\langle a_{1}^{\dagger}, a_{1}\right\rangle+\left\langle a_{1}, a_{1}^{\dagger}\right\rangle+\left\langle a_{2}^{\dagger}, a_{2}\right\rangle+\left\langle a_{2}, a_{2}^{\dagger}\right\rangle \\
& +2\left(\left\langle a_{1}, a_{2}\right\rangle+\left\langle a_{1}^{\dagger}, a_{2}^{\dagger}\right\rangle\right),
\end{aligned}
$$

where we used the notation $\langle a, b\rangle=\langle a b\rangle-\langle a\rangle\langle b\rangle$. In order to study the entanglement, we calculate the time evolution of various moments involved in the quantity $(\Delta \hat{u})^{2}+(\Delta \hat{v})^{2}$ using the equations of motion given in Appendix B. The ana-

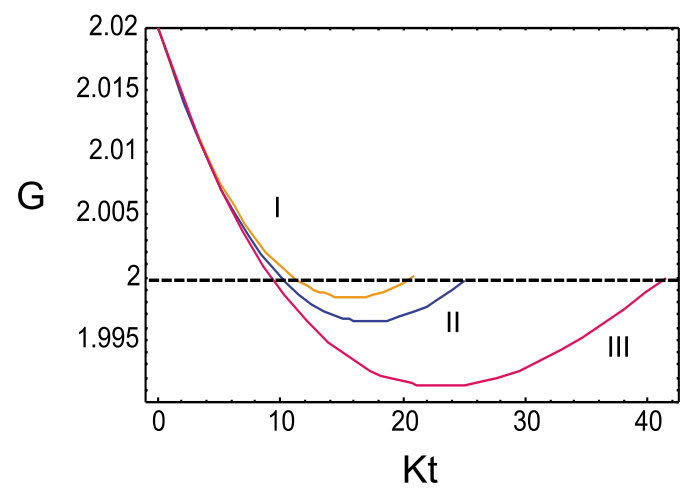

FIG. 5. (Color online) Time development of $G \equiv(\Delta \hat{u})^{2}+(\Delta \hat{v})^{2}$ in terms of the normalized interaction time $K t$ for a squeezed vacuum state in mode 1 and a coherent state in mode 2 with $r$ $=0.1$ and $\alpha=10$. Here curves I, II, and III represent the results for $\Omega=1400,1500$, and $2000 \mathrm{kHz}$, espectively. All the other parameters are the same as in Fig. 2.

lytical solutions are, as before, rather complicated; therefore, we again present the results of our numerical simulations.

In Fig. 5, we show the plots of the time development of $G=(\Delta \hat{u})^{2}+(\Delta \hat{v})^{2}$ as a function of the dimensionless interaction time $K t$. The initial state of the field in mode 1 is a squeezed vacuum state, while the mode 2 is in a coherent state. Here curves I, II, and III correspond to $\Omega=1400 \mathrm{kHz}$, $1500 \mathrm{kHz}$, and $2000 \mathrm{kHz}$, respectively. All the other parameters are the same as in Fig. 2. The results show that the parameter $\mathrm{G}$ evolves with time and becomes less then 2, showing that the two modes, which were initially in a product state, become entangled. However, after some time the parameter $\mathrm{G}$ becomes equal to 2 , and the entanglement condition is no longer satisfied. This is due to the presence of dissipation in the system. It is clear from curves I, II, and III that the time interval for the two modes to remain entangled increases as the amplitude of the Rabi frequency $\Omega$ of the driving field is increased. A comparison of the results shown by curve II in Fig. 5 with those shown by curve $C$ in Fig. 2 (which corresponds to the same set of parameters) shows that the two entanglement criteria give different time intervals for which the two modes remain entangled. For example, the Hillery-Zubairy criterion shows that the two modes become entangled as soon as the system evolves, whereas according to the Simon-DGCZ criterion, the system is not entangled unless the value of the normalized interaction time $K t$ is approximately equal to 13 .

\section{CONCLUSION}

In conclusion, we have studied the generation of entangled states in a quantum beat laser in the presence of cavity losses using the Hillery-Zubairy, and the SimonDGCZ criteria. We considered different initial states of the field in the two modes, for example, a squeezed vacuum state in mode 1 and a coherent state in mode 2, and a photon number state in mode 1 and a vacuum state in mode 2 . Our results show that the initial states, which are in a product state, evolve into an entangled state. The time for which the two modes remain entangled depends upon the strength of 
the Rabi frequency of the classical driving field, and this time can be increased by increasing it. Our results also show that the two criteria give different time intervals for the entanglement, which is quite interesting. This indicates that here both the criteria provide only sufficient conditions for the entanglement. If they had provided the necessary and sufficient conditions then the time intervals for the entanglement determined by the two criteria should be the same. It may be mentioned that in some very specific cases these criteria can lead to necessary and sufficient conditions for entanglement. We also present results for average photon number in the two modes, which exhibits damped oscillations. The temporal behavior of the average photon numbers gives an insight into the increase in the entanglement time due to the increase in the amplitude of the Rabi frequency.

\section{ACKNOWLEDGMENTS}

The authors would like to thank COMSTECH for their support. We would like to thank Sajid Qamar for some fruitful discussion. This research is partially supported by the Qatar Natonal Research Foundation (QNRF).

\section{APPENDIX A}

The coefficients $\alpha_{11}, \alpha_{12}, \alpha_{21}$, and $\alpha_{22}$ in Eq. (4) are given by the following:

$$
\begin{aligned}
& \alpha_{11}= \frac{g^{2} r_{a}}{2 \gamma\left(\gamma^{2}+\Omega^{2}\right)}\left(\frac{\left(2 \gamma^{2}+\Omega^{2}+i \Omega \gamma\right)[\gamma-i(\Delta-\Omega / 2)]}{\left[\gamma^{2}+(\Delta-\Omega / 2)^{2}\right]}\right. \\
&\left.+\frac{\left(2 \gamma^{2}+\Omega^{2}-i \Omega \gamma\right)[\gamma-i(\Delta+\Omega / 2)]}{\left[\gamma^{2}+(\Delta+\Omega / 2)^{2}\right]}\right), \\
& \alpha_{12}= \frac{g^{2} r_{a} \Omega}{2 \gamma\left(\gamma^{2}+\Omega^{2}\right)}\left(\frac{[\gamma-i(\Delta-\Omega / 2)]}{\left[\gamma^{2}+(\Delta-\Omega / 2)^{2}\right]}(\Omega-i \gamma)\right. \\
&\left.-\frac{[\gamma-i(\Delta+\Omega / 2)]}{\left[\gamma^{2}+(\Delta+\Omega / 2)^{2}\right]}(\Omega+i \gamma)\right), \\
& \alpha_{21}= \frac{g^{2} r_{a}}{2 \gamma\left(\gamma^{2}+\Omega^{2}\right)}\left(\frac{\left(2 \gamma^{2}+\Omega^{2}+i \Omega \gamma\right)[\gamma-i(\Delta-\Omega / 2)]}{\left[\gamma^{2}+(\Delta-\Omega / 2)^{2}\right]}\right. \\
&-\left.\frac{\left(2 \gamma^{2}+\Omega^{2}-i \Omega \gamma\right)[\gamma-i(\Delta+\Omega / 2)]}{\left[\gamma^{2}+(\Delta+\Omega / 2)^{2}\right]}\right), \\
& \alpha_{22}= \frac{g^{2} r_{a} \Omega}{2 \gamma\left(\gamma^{2}+\Omega^{2}\right)}\left(\frac{[\gamma-i(\Delta-\Omega / 2)]}{\left[\gamma^{2}+(\Delta-\Omega / 2)^{2}\right]}(\Omega-i \gamma)\right. \\
&\left.+\frac{\left[\gamma^{2}+(\Delta+\Omega / 2)\right]}{\left.(\Delta / 2)^{2}\right]}(\Omega+i \gamma)\right) .
\end{aligned}
$$

\section{APPENDIX B}

The equations of motion for various moments required to study the generation of entanglement using a quantum beat laser can easily be obtained from Eq. (4). For a suitable choice of the phase $\Phi=\pi / 2$ associated with the classical driving field, the following sets of coupled equations for various moments are obtained:

$$
\begin{gathered}
\frac{d}{d t}\left\langle a_{1}\right\rangle=\frac{1}{2}\left(\alpha_{11}-2 \kappa_{1}\right)\left\langle a_{1}\right\rangle+\frac{i}{2} \alpha_{12}\left\langle a_{2}\right\rangle, \\
\frac{d}{d t}\left\langle a_{2}\right\rangle=\frac{1}{2}\left(\alpha_{22}-2 \kappa_{2}\right)\left\langle a_{2}\right\rangle-\frac{i}{2} \alpha_{21}\left\langle a_{1}\right\rangle, \\
\frac{d}{d t}\left\langle a_{1} a_{2}^{\dagger}\right\rangle=\frac{1}{2}\left[\left(\alpha_{11}+\alpha_{22}^{*}\right)-\left(\kappa_{1}+\kappa_{2}\right)\right]\left\langle a_{1} a_{2}^{\dagger}\right\rangle+\frac{i}{2}\left(\alpha_{21}^{*}\left\langle a_{1}^{\dagger} a_{1}\right\rangle\right. \\
\left.+\alpha_{12}\left\langle a_{2}^{\dagger} a_{2}\right\rangle\right)+\frac{i}{2}\left(\alpha_{21}^{*}+\alpha_{12}\right),
\end{gathered}
$$

$$
\begin{aligned}
\frac{d}{d t}\left\langle a_{1}^{\dagger} a_{2}\right\rangle= & \frac{1}{2}\left[\left(\alpha_{11}^{*}+\alpha_{22}\right)-\left(\kappa_{1}+\kappa_{2}\right)\right]\left\langle a_{1}^{\dagger} a_{2}\right\rangle-\frac{i}{2}\left(\alpha_{21}\left\langle a_{1}^{\dagger} a_{1}\right\rangle\right. \\
& \left.+\alpha_{12}^{*}\left\langle a_{2}^{\dagger} a_{2}\right\rangle\right)-\frac{i}{2}\left(\alpha_{21}+\alpha_{12}^{*}\right), \\
\frac{d}{d t}\left\langle N_{1}\right\rangle= & \frac{1}{2}\left[\left(\alpha_{11}+\alpha_{11}^{*}\right)-2 \kappa_{1}\right]\left\langle N_{1}\right\rangle+\frac{i}{2}\left(\alpha_{12}\left\langle a_{1}^{\dagger} a_{2}\right\rangle\right. \\
& \left.-\alpha_{12}^{*}\left\langle a_{1} a_{2}^{\dagger}\right\rangle\right)+\frac{1}{2}\left(\alpha_{11}+\alpha_{11}^{*}\right),
\end{aligned}
$$

$$
\begin{aligned}
\frac{d}{d t}\left\langle N_{2}\right\rangle= & \frac{1}{2}\left[\left(\alpha_{22}+\alpha_{22}^{*}\right)-2 \kappa_{2}\right]\left\langle N_{2}\right\rangle+\frac{i}{2}\left(\alpha_{21}^{*}\left\langle a_{1}^{\dagger} a_{2}\right\rangle\right. \\
& \left.-\alpha_{21}\left\langle a_{1} a_{2}^{\dagger}\right\rangle\right)+\frac{1}{2}\left(\alpha_{22}+\alpha_{22}^{*}\right),
\end{aligned}
$$

$$
\begin{aligned}
\frac{d}{d t}\left\langle a_{1} a_{2}\right\rangle= & \left(\frac{1}{2}\left(\alpha_{11}+\alpha_{22}\right)-\left(\kappa_{1}+\kappa_{2}\right)\right)\left\langle a_{1} a_{2}\right\rangle \\
& +\frac{i}{2} \alpha_{12}\left\langle a_{2} a_{2}\right\rangle-\frac{i}{2} \alpha_{21}\left\langle a_{1} a_{1}\right\rangle
\end{aligned}
$$

$$
\frac{d}{d t}\left\langle a_{1} a_{1}\right\rangle=\left(\alpha_{11}-2 \kappa_{1}\right)\left\langle a_{1} a_{1}\right\rangle+i \alpha_{12}\left\langle a_{1} a_{2}\right\rangle
$$

$$
\frac{d}{d t}\left\langle a_{2} a_{2}\right\rangle=\left(\alpha_{22}-2 \kappa_{2}\right)\left\langle a_{2} a_{2}\right\rangle-i \alpha_{21}\left\langle a_{1} a_{2}\right\rangle
$$

$$
\begin{aligned}
\frac{d}{d t}\left\langle N_{1} N_{2}\right\rangle= & \left(\frac{1}{2}\left(\alpha_{11}+\alpha_{11}^{*}+\alpha_{22}+\alpha_{22}^{*}\right)-2\left(\kappa_{1}+\kappa_{2}\right)\right)\left\langle N_{1} N_{2}\right\rangle \\
& +\frac{1}{2}\left(\alpha_{22}+\alpha_{22}^{*}\right)\left\langle N_{1}\right\rangle+\frac{1}{2}\left(\alpha_{11}+\alpha_{11}^{*}\right)\left\langle N_{2}\right\rangle \\
& +\frac{i}{2}\left(-\alpha_{21}\left\langle N_{1} a_{1} a_{2}^{\dagger}\right\rangle+\alpha_{21}^{*}\left\langle N_{1} a_{1}^{\dagger} a_{2}\right\rangle\right) \\
& +\frac{i}{2}\left(-\alpha_{12}^{*}\left\langle a_{1} a_{2}^{\dagger} N_{2}\right\rangle+\alpha_{12}\left\langle a_{1}^{\dagger} a_{2} N_{2}\right\rangle\right) \\
& -\frac{i}{2}\left(\alpha_{12}^{*}+\alpha_{21}\right)\left\langle a_{1} a_{2}^{\dagger}\right\rangle,
\end{aligned}
$$




$$
\begin{aligned}
\frac{d}{d t}\left\langle N_{1} a_{1}^{\dagger} a_{2}\right\rangle= & \left(\frac{1}{2}\left(2 \alpha_{11}^{*}+\alpha_{11}+\alpha_{22}\right)-\left(3 \kappa_{1}+\kappa_{2}\right)\right)\left\langle N_{1} a_{1}^{\dagger} a_{2}\right\rangle \\
& +\left(\frac{1}{2}\left(\alpha_{11}+\alpha_{11}^{*}\right)+2 \kappa_{1}\right)\left\langle a_{1}^{\dagger} a_{2}\right\rangle+\frac{1}{2} \alpha_{12} i\left\langle a_{1}^{\dagger} a_{2}^{2}\right\rangle \\
& -i\left(\alpha_{12}^{*}\left\langle N_{1} N_{2}\right\rangle+\frac{1}{2} \alpha_{21}\left\langle N_{1}^{2}\right\rangle+\frac{1}{2}\left(\alpha_{12}^{*}+\alpha_{21}\right)\left\langle N_{1}\right\rangle\right. \\
& \left.+\frac{1}{2} \alpha_{12}^{*}\left\langle N_{2}\right\rangle+\frac{1}{2}\left(\alpha_{12}^{*}+\alpha_{21}\right)\right), \\
\frac{d}{d t}\left\langle N_{1} a_{1} a_{2}^{\dagger}\right\rangle= & \left.\frac{1}{2}\left(\alpha_{11}^{*}+2 \alpha_{11}+\alpha_{22}^{*}\right)-\left(3 \kappa_{1}+\kappa_{2}\right)\right)\left\langle N_{1} a_{1} a_{2}^{\dagger}\right\rangle \\
& +\left(\alpha_{11}+\alpha_{11}^{*}\right)\left\langle a_{1} a_{2}^{\dagger}\right\rangle-\frac{i}{2} \alpha_{12}^{*}\left\langle a_{1}^{2} a_{2}^{\dagger 2}\right\rangle \\
& -i\left(\alpha_{12}\left\langle N_{1} N_{2}\right\rangle+\frac{1}{2} \alpha_{21}^{*}\left\langle N_{1}^{2}\right\rangle\right. \\
& \left.+\frac{1}{2}\left(2 \alpha_{12}+\alpha_{21}^{*}\right)\left\langle N_{1}\right\rangle\right), \\
& -i\left(\alpha_{21}\left\langle N_{1} N_{2}\right\rangle+\frac{1}{2} \alpha_{12}^{*}\left\langle N_{2}\right\rangle+\left(\alpha_{12}^{*}+\alpha_{21}\right)\left\langle N_{2}\right\rangle\right. \\
& +\left(\frac{1}{2}\left(\alpha_{22}+\alpha_{22}^{*}\right)+2 \kappa_{2}\right)\left\langle a_{1}^{\dagger} a_{2}\right\rangle+\frac{i}{2} \alpha_{21}^{*}\left\langle a_{1}^{\dagger} a_{2}^{2}\right\rangle \\
& \left.+\frac{1}{2}\left(2 \alpha_{21}^{*}+\alpha_{12}\right)\left\langle N_{2}\right\rangle\right), \\
& +\left(\alpha_{21}^{*}\left\langle N_{1} N_{2}\right\rangle+\frac{1}{2} \alpha_{12}\left\langle N_{2}^{2}\right\rangle\right. \\
& +\left(\alpha_{22}+\alpha_{22}^{*}\right)\left\langle a_{1} a_{2}^{\dagger}\right\rangle-\frac{i}{2} \alpha_{21}\left\langle a_{1}^{2} a_{2}^{\dagger 2}\right\rangle \\
\left\langle a_{1} a_{2}^{\dagger} N_{2}\right\rangle= & \left.\frac{1}{2}\left(\alpha_{11}^{*}+\alpha_{22}^{*}+2 \alpha_{22}\right)-\left(\kappa_{1}+3 \kappa_{2}\right)\right)\left\langle a_{1}^{\dagger} a_{2} N_{2}\right\rangle \\
& (\mathrm{B} 12) \\
&
\end{aligned}
$$

$$
\begin{aligned}
\frac{d}{d t}\left\langle N_{2}^{2}\right\rangle= & {\left[\left(\alpha_{22}+\alpha_{22}^{*}\right)-4 \kappa_{2}\right]\left\langle N_{2}^{2}\right\rangle+\left(\frac{3}{2}\left(\alpha_{22}+\alpha_{22}^{*}\right)+2 \kappa_{2}\right)\left\langle N_{2}\right\rangle } \\
& +\frac{1}{2}\left(\alpha_{22}+\alpha_{22}^{*}\right)+\frac{i}{2} \alpha_{21}^{*}\left(2\left\langle a_{1}^{\dagger} a_{2} N_{2}\right\rangle-\left\langle a_{1}^{\dagger} a_{2}\right\rangle\right) \\
& +\frac{i}{2} \alpha_{21}\left(2\left\langle a_{1} a_{2}^{\dagger} N_{2}\right\rangle+\left\langle a_{1} a_{2}^{\dagger}\right\rangle\right) .
\end{aligned}
$$

where the coefficients $\alpha_{11}, \alpha_{22}, \alpha_{12}$, and $\alpha_{21}$ are listed in Appendix A.

$$
\frac{d}{d t}\left\langle a_{1}^{\dagger} a_{2}^{2}\right\rangle=\left[\left(\alpha_{11}^{*}+\alpha_{22}\right)-2\left(\kappa_{1}+\kappa_{2}\right)\right]\left\langle a_{1}^{\dagger} a_{2}^{2}\right\rangle-i\left[\alpha_{12}^{*}\left\langle a_{1}^{\dagger} a_{2} N_{2}\right\rangle\right.
$$$$
\frac{d}{d t}\left\langle a_{1}^{2} a_{2}^{\dagger 2}\right\rangle=\left[\left(\alpha_{11}+\alpha_{22}^{*}\right)-2\left(\kappa_{1}+\kappa_{2}\right)\right]\left\langle a_{1}^{2} a_{2}^{\dagger 2}\right\rangle+i\left[\alpha_{21}^{*}\left\langle N_{1} a_{1} a_{2}^{\dagger}\right\rangle\right.
$$$$
\left.+\alpha_{12}\left\langle a_{1} a_{2}^{\dagger} N_{2}\right\rangle+2\left(\alpha_{21}^{*}+\alpha_{12}\right)\left\langle a_{1} a_{2}^{\dagger}\right\rangle\right]
$$$$
\frac{d}{d t}\left\langle N_{1}^{2}\right\rangle=\left[\left(\alpha_{11}+\alpha_{11}^{*}\right)-4 \kappa_{1}\right]\left\langle N_{1}^{2}\right\rangle+\left(\frac{3}{2}\left(\alpha_{11}+\alpha_{11}^{*}\right)+2 \kappa_{1}\right)\left\langle N_{1}\right\rangle
$$$$
+\frac{1}{2}\left(\alpha_{11}+\alpha_{11}^{*}\right)+\frac{i}{2} \alpha_{12}\left(2\left\langle N_{1} a_{1}^{\dagger} a_{2}\right\rangle-\left\langle a_{1}^{\dagger} a_{2}\right\rangle\right)
$$$$
-\frac{i}{2} \alpha_{12}^{*}\left(2\left\langle N_{1} a_{1} a_{2}^{\dagger}\right\rangle+\left\langle a_{1} a_{2}^{\dagger}\right\rangle\right)
$$

[1] A. Furusawa, J. L. Sorensen, S. L. Braunstein, C. A. Fuchs, H. J. Kimble, and E. S. Polzik, Science 282, 706 (1998).

[2] Quantum Information with Continuous Variable, edited by S.L. Braunstein and A. K. Pati (Kluwer, Dordrecht, 2003).

[3] S. L. Braunstein and H. J. Kimble, Phys. Rev. Lett. 80, 869 (1998).

[4] J. Hald, J. L. Sorensen, C. Schori, and E. S. Polzik, Phys. Rev. Lett. 83, 1319 (1999); B. Julsgaard, A. Kozhekin, and E. S. Polzik, Nature (London) 413, 400 (2001).

[5] A. S. Villar, L. S. Cruz, K. N. Cassemiro, M. Martinelli, and P. Nussenzveig, Phys. Rev. Lett. 95, 243603 (2005).

[6] C. Simon and D. Bouwmeester, Phys. Rev. Lett. 91, 053601 
022305 (2005).

[13] K. Ahmed, H. Xiong, and M. S. Zubairy, Opt. Commun. 262, 129 (2006).

[14] S. Qamar, H. Xiong, and M. S. Zubairy, Phys. Rev. A 75, 062305 (2007).

[15] S. Y. Lee, S. Qamar, H. W. Lee, and M. S. Zubairy (unpublished).

[16] L. M. Duan, G. Giedke, J. I. Cirac, and P. Zoller, Phys. Rev. Lett. 84, 2722 (2000).

[17] M. Hillery and M. S. Zubairy, Phys. Rev. Lett. 96, 050503 (2006), and references therein.

[18] M. O. Scully and M. S. Zubairy, Phys. Rev. A 35, 752 (1987).

[19] M. Ohtsu and K.-Y. Liou, Appl. Phys. Lett. 52, 10 (1988); M. P. Winters, J. L. Hall, and P. E. Toschek, Phys. Rev. Lett. 65, 3116 (1990).

[20] R. Simon, Phys. Rev. Lett. 84, 2726 (2000).
[21] E. Shchukin and W. Vogel, Phys. Rev. Lett. 95, 230502 (2005).

[22] G. S. Agarwal and A. Biswas, New J. Phys. 7, 211 (2005).

[23] A. Peres, Phys. Rev. Lett. 77, 1413 (1996).

[24] M. Horodecki, P. Horodecki, and R. Horodecki, Phys. Rev. Lett. 93, 170503 (2004).

[25] M. Hillery and M. S. Zubairy, Phys. Rev. A 74, 032333 (2006).

[26] H. P. Yuen and J. H. Shapiro, IEEE Trans. Inf. Theory 26, 78 (1980).

[27] D. Meschede, H. Walther, and G. Muller, Phys. Rev. Lett. 54, 551 (1985); G. Raqithel, C. Wagner, H. Walther, L. M. Narducci, and M. O. Scully, Adv. At., Mol., Opt. Phys. 33 Suppl. 2, 57 (1994).

[28] J. M. Raimond, M. Brune, and S. Haroche, Rev. Mod. Phys. 73, 565 (2001). 